\title{
Perspectives
}

\section{Effect of Pharmacogenetics on Medicine}

\author{
Wendell W. Weber* \\ Department of Pharmacology, University of Michigan, Ann Arbor, Michigan
}

\begin{abstract}
Pharmacogenetics is moving rapidly to assemble a large set of polymorphisms that define the influence of genetic diversity on human drug response. Scientific and technological advances of the last 10 years have led to new approaches to the discovery of genetic drug susceptibility loci, the development of high-tech analytical strategies for drug susceptibility profiling, and a flood of new gene discoveries in the area of receptors and receptor polymor-
\end{abstract}

phisms. Extension and refinement of our knowledge of human genetic diversity is essential to the use of drugs in more of an individualized manner and to the discovery of better therapies, but knowledge of the functional consequences of this diversity, the next great challenge in pharmacogenetics, provides the best chance to profit from this diversity. Environ. Mol. Mutagen. 37: 179-184, 2001. @ 2001 Wiley-Liss, Inc.

Key words: pharmacogenetics; pharmacokinetics; pharmacogenomics; genetic profile; xenobiotics; receptors; Invader technology

\section{INTRODUCTION}

Physicians rely on drugs as therapeutic agents because of the characteristic, reproducible effects they exert on persons. Unfortunately, for those who fail to respond as expected, or suffer toxicity from these agents, the effects are all too apparent. Researchers discovered decades ago that genetics affects person-to-person variation in responses to drugs and other xenobiotics. Pharmacogenetics, a field primarily concerned with understanding how genetic diversity within populations influences drug effectiveness and toxicity, provides the experimental framework for dissecting the causes of this variation. Knowing the intrinsic variation in the human genetic anatomy and its functional consequences will permit genetic profiles for individuals to be constructed that will enable physicians to identify persons likely to be susceptible to unexpected drug responses. It is hoped and expected that drug susceptibility profiles can be used to select drugs better fitted to safe, effective therapy and to design better therapies. As a consequence, pharmacogenetics is experiencing a period of rapid growth and redefinition.

This communication describes the present state of pharmacogenetics and highlights some of the challenges of the near future that face pharmacogenetics. Because pharmacogenetics is mistakenly perceived in some circles as an outgrowth of the biological revolution accompanying recombinant DNA technology, we begin with a brief history of the field.

\section{ORIGIN AND DEVELOPMENT OF PHARMACOGENETICS}

Pharmacogenetics has a rich history with several roots. Up to about 1990, the emergence and growth of the field falls neatly into three periods, the first of which began about 150 years ago, the second began about 1910, and the third about 1950.

Around 1850 and extending to about 1910, physiological chemists, mainly in European laboratories, found that humans were capable of metabolizing most drugs and exogenous chemicals they ingested. In the 1860s, Mendel discovered the fundamental laws of heredity, but his findings were unappreciated until their rediscovery by others around 1900 . And in the 1870s, Ehrlich and Langley inferred the existence of drug receptor molecules to account for the specificity of drug action on tissues. Conceptually, the origin of pharmacogenetics is closely tied to these three discoveries.

During the second period from 1910 to about 1950, the importance of these discoveries began to emerge when Archibald Garrod and William Bateson in England and Lucien Cuenot in France suggested that the genetic material played a crucial part in directing the chemical transformations described by physiological chemists. Garrod was first to realize that enzymes could act as detoxifiers of exogenous substances; he believed that the poisonous effects of drugs experienced by certain persons was likely the result of a

Presented at the symposium on Pharmacogenomics at the 31st Annual Meeting of the Environmental Mutagen Society, April 9-13, 2000, New Orleans, LA.

*Correspondence to: Wendell W. Weber, University of Michigan, Department of Pharmacology, 1301b MSRB III, 1150 W. Medical Center Drive, Ann Arbor, MI 48109-0632. E-mail: wwweber@umich.edu

Received 1 December 2000; accepted in final form 3 January 2001 
failure of their enzymes to detoxify those substances. He stressed these ideas in writings and teachings until his death in the 1930s as evidence gradually accumulated for the importance of chemical individuality of humans. During the 1920s and 1930s, the traits of "odor blindness" and "taste blindness" were recognized, and detailed study of taste blindness revealed the high specificity, inheritance, and ethnic variability that characterize human responsiveness to chemicals. Another decade would pass, however, before we learned from studies of sickle cell anemia and sickle cell trait (1948-1950) that these disorders were caused by an aberrant form of protein (hemoglobin S), and that the responses of persons to the environment reflected the properties of proteins they synthesize [reviewed in Weber, 1997].

The third period begins around 1950 and extends until about 1990. During this period the importance of genetic variation to the safe use of drugs was firmly established. Early in this period, several breakthroughs occurred in short order, prompting biologists to take a more genetic approach to their research. Discovery of the double helix of DNA defined the molecular basis of heredity, human chromosomes were visualized and enumerated, and protein polymorphism was recognized as a phenomenon of major biological significance. New techniques for separating drugs and their metabolites and a desire to get at the causes of unexpected drug responses enabled pharmacologists with a biochemical bent to describe heritable patterns of metabolism and response for several drugs (1953-1956).

About 100 monogenic, polymorphic traits of pharmacogenetic interest were identified by about 1990. The lion's share of these were found to be attributed to polymorphic isoforms of the drug-metabolizing enzymes, affirming again and again Garrod's idea of enzymes as detoxifiers of environmental chemicals. Rigorous pharmacological and genetic characterization of these traits were chiefly responsible for shaping the initial development of the field and establishing pharmacogenetics as an experimental science.

\section{TRANSITION TO THE FUTURE, 1990-2000}

The advent of molecular genetics during the 1980s radically altered the way of doing modern biology. Cloning, sequencing, and site-directed mutagenesis became the norm for studying the organization and composition of genes. Widespread application of recombinant DNA techniques quickened the pace of investigation and enabled the identity, chromosomal location, and characteristics of genes for virtually every known pharmacogenetic trait that had been determined. To keep everyone abreast of a rapidly expanding and intimidating body of evidence and of new directions in research, a plethora of educational programs, workshops, and conferences were produced to create awareness of these advances. Moreover, efforts to find new ways to catalog the new knowledge about genes, compare variations in their expression, study their interactions, and identify their func- tions have encouraged scientists in academia and industry to form partnerships for the betterment of testing pharmacogenetic initiatives.

\section{Novel Approaches to the Discovery of Drug Susceptibility Loci}

One of the first changes to occur concerned the way to seek genes that might alter person-to-person susceptibility to drugs. Traditionally, pharmacogeneticists had relied on patterns of inheritance of phenotypic criteria to identify genetic factors implicated in an altered drug response. The susceptible phenotype usually had physiologic, biochemical, pharmacologic, or toxicologic features that could lead to inferences about the identity of the protein and even the gene and allelic variants responsible for the phenotype. For instance, in the 1950s, to explain the susceptibility of persons to succinylcholine sensitivity, Kalow inferred the existence of a genetic variant of serum cholinesterase from the presence of a serum cholinesterase with unusual resistance to inhibition by dibucaine. About the same time, to explain the susceptibility of persons to isoniazid-induced nerve damage, the existence of a genetic variant of acetyltransferase was inferred from low levels of acetylated isoniazid metabolites in the urine of patients administered the drug [see references in Weber, 1987, Chapters 2 and 3].

With the advent of recombinant DNA technology, however, the genes for serum cholinesterase, acetyltransferase, as well as for many other monogenic traits were cloned and sequenced, and the existence of variant genes demonstrated. Thus the connection could now be directly established between a susceptible phenotype and its genotype that heretofore could only be inferred. That is, by starting with the gene sequence and proceeding through expression of the protein, the susceptible biochemical or physiological phenotype was determined.

Nowadays, because a gene sequence and its chromosomal location can be determined relatively easily, the anatomy of a gene and its position may be known before its function or the phenotype it determines. Hence, use of the combined information on function and position has become increasingly common as a means of gene identification [Ballabio, 1993].

\section{Invention of Techniques to Facilitate Drug Discovery}

A number of techniques have been invented for identifying and scoring polymorphisms [see Box 1, Schafer and Hawkins, 1998]. Where the primary focus for research and diagnosis has been on simple, high-throughput analysis, techniques such as the DNA microarray and Invader technologies are outstanding.

Increasing demand for analytical techniques that could accommodate a high density of information to sequence, map, and identify human genes led to the development of 
DNA microarrays. DNA microarrays, like all other DNA hybridization analytical technologies, are modifications of the "Southern blot" devised by Edwin Southern in 1975 [Weber and Cronin, 2000]. The Southern blot uses the specificity of DNA restriction endonucleases combined with gel electrophoresis to separate and identify DNA fragments. Southern's method quickly evolved into several other techniques. First, the Northern blot (1977) and then DNA fingerprinting (1985) were devised. In 1989, "dot" blots and "slot" blots, which used test samples of DNA as the immobilized species for analysis of DNA fragments, were devised. They were faster and could accommodate smaller DNA samples than previous techniques. Later, polymerase chain reaction (PCR) permitted the development of the "reverse dot blot," in which DNA probes, instead of DNA test samples, became the immobilized species (1991-1994). In 1994, Southern, again at the forefront of advances, first described the in situ synthesis of an oligonucleotide array on solid supports [reviewed in Weber and Cronin, 2000]. Application of his method and its modifications led to microarrays, which consist of segments of complementary DNA (cDNA) or of expressed sequence tags immobilized on a solid support.

Despite remarkable innovation and rapid technical improvement since then, improved fabrication, reduced costs, and enhanced robustness and reliability must yet be achieved before microarrays reach their full potential as analytical tools. There are also significant challenges in storage and handling of vast amounts of information that microarrays have created. New concepts are also needed to relate large complex sets of genetic data to clinical phenotypes. Hence, predictive testing is currently available for only a very few, if any, applications based on DNA microarray profiling.

Microarray analysis allows all of the genes in a system to be monitored at once instead of one gene at a time, thus enabling the collection of large amounts of genetic data from comparatively few experiments. Currently, microarray applications span many aspects of molecular biological investigation by helping to diagnose diseases, illuminate developmental and physiological pathways, measure and compare perturbations in gene activities and patterns of gene expression in cancer and other pathological states, and screen drug candidates for new therapies.

With respect to pharmacogenetics, microarrays are used mainly as research tools to learn the most effective ways of gathering information for the construction of genetic profiles of susceptibility to unexpected responses to drugs and other xenobiotics.

The Invader system of techniques that has been developed more recently provides another analytical method for investigating alterations of the human genome. The Invader system finds its origin in the properties of the cleavase enzymes derived from structure-specific endonucleases that are essential for DNA replication, recombination, and repair
[Kwiatkowski et al., 1999 and references therein]. Invader techniques can use genomic DNA directly from small blood volumes, or cell lysates, for genotyping and they can discriminate mutant from wild-type genotypes in mixed populations at very low ratios (1/1000 or lower) of mutant/wild type. In the case of gene expression analysis, the technique can quantitate closely related messages within the same sample. Additionally, this technology is adaptable for use in large-scale analysis of single-nucleotide polymorphisms (SNPs) that use high-throughput screening methods of detection such as the fluorescence-based (FRET) system and matrix-assisted laser desorption/ionization time of flight (MALDI/TOF) mass spectrometry. Applications of the Invader system to factor V Leiden, factor II (prothrombin), cystic fibrosis, and apolipoprotein $\mathrm{E}$, and to measurement of mRNA levels of ubiquitin demonstrate its validity for mutation detection, SNP profiling, and gene expression analysis. The Invader technology is quite promising as a new tool for research and clinical application in pharmacogenetics.

\section{The Flood of New Gene Discoveries}

Among all the genes whose structure and function have been elucidated during the last decade, the genes for receptors and receptor polymorphisms stand out. Cell surface receptors are the primary focus of the following discussion not only because they account for the response of cells to most drugs, biogenic amines, protein and polypeptide hormones, autocoids, and neurotransmitters, but also because susceptibility profiles that are associated with several prototypic polymorphisms of these receptors have been identified [Weber, 2001].

The association of polymorphic variants of ion channels to cardiovascular disease yields several important examples. For $\mathrm{K}^{+}$channels [Curran, 1998], genetic and physiologic studies indicate that mutations in four loci are implicated in susceptibility to the long QT (LQT) syndrome (LQT 1, 2, 5, and 6 ). These variants are associated with cardiac arrhythmias. Homozygosity of the KVLQT1 variant, identified in 1991, causes the Jervell Lange-Nielsen syndrome (LQT plus deafness), whereas heterozygosity causes the RomanoWard syndrome (LQT only), the most common inherited form of LQTs. Mutation of the $\mathrm{Na}^{+}$channel $(S C N 5 \mathrm{~A}=$ LQT3) is also associated with cardiac arrhythmias. Knowledge of the LQT genotype that results in a specific clinical phenotype may provide a basis for gene-specific drug therapy. For example, potassium supplementation or potassium channel openers are useful in treating patients with potassium channel defects, whereas sodium channel blockers (e.g., mexilitene) may be more effective in treating patients with $L Q T 3$.

The $\beta_{2}$-adrenergic receptor, a prototypic example of Gprotein receptors [Lefkowitz, 2000], has been targeted for investigation of susceptibility to asthma since the betaadrenergic receptor theory of atopy was proposed 30 years 


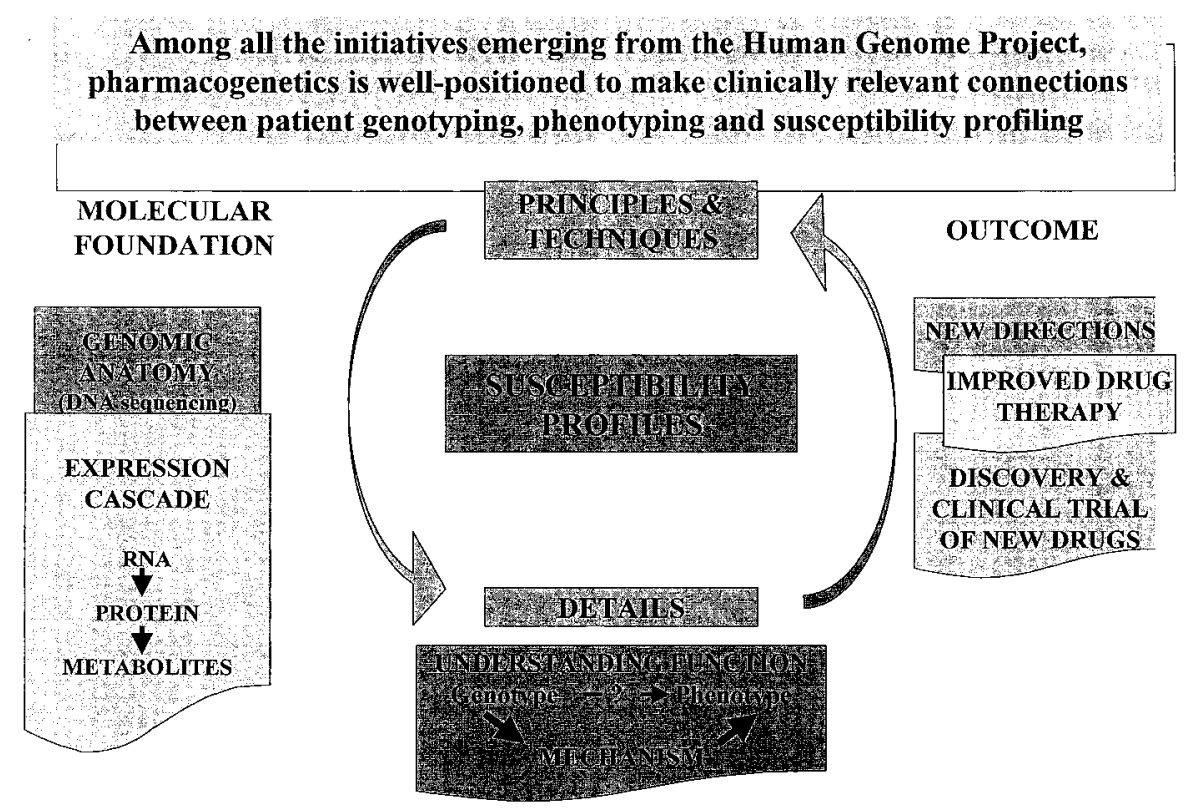

Fig. 1. Pharmacogenetics in the year 2000 .

ago. The receptor was cloned, sequenced, and mapped in 1987. Initial molecular studies revealed a total of nine naturally occurring single-nucleotide polymorphisms (SNPs). Three SNPs resulted in changes in amino acids 16 and 27 of the receptor, which altered its abundance and function, but a more recent study identifies a total of 13 SNPs organized into 12 haplotypes [Drysdale et al., 2000]. Studies in Caucasian, African-American, Asian, and Hispanic-Latino populations revealed more than 20 -fold differences among the frequencies of the four major haplotypes in these populations. The responses to bronchodilator therapy with albuterol of individuals with the five most common $\beta_{2}$-adrenergic receptor haplotypes were determined in vivo by haplotype pair; the mean responses varied by more than twofold. Although the mean responses were significantly related to haplotype pair, they were not related to individual SNPs. This study is among the first to indicate that interactions between SNPs within a haplotype can affect biologic and therapeutic phenotype, and may have prognostic significance in assessing individual variation in drug response.

\section{PROFITING FROM HUMAN GENETIC DIVERSITY}

The use of genetic information to predict human responsiveness to pharmaceutical agents and other exogenous chemicals is the foremost purpose of pharmacogenetics. Physicians know that a patient with a genetic drug susceptibility is no less treatable than many other patients they manage by restricting access to, or by replacing and/or removing, the offending substance from the environment. It follows that, if treatment is guided by profiles for susceptibility of individuals to specific drugs, the occurrence of unexpected responses among genetically susceptible persons might be averted.

Pharmacogenetics is well positioned to synthesize such profiles by virtue of having an extensive base of reliable data about monogenic traits attributable to drug-metabolizing enzyme polymorphisms and numerous other polymorphisms. Indeed, these traits are remarkable prognosticators of therapeutic failure of, or unexpected responses to, xenobotics, although as these observations are examined in greater depth, it is becoming clear that few, if any, phenotypic outcomes will be reliably predicted from genetic analyses at a single locus. True predictive pharmacogenetic testing relies on having broad individual genotyping capability and integrated rational therapeutic strategies for disease, guided by genetic predisposition and risk profiles. The full potential for predictive pharmacogenetic testing will be realized only when these goals as well as bioinformatics to analyze large data sets are also fully developed [Weber and Cronin, 2000]. Nevertheless, we expect that extension and refinement of the body of evidence mentioned earlier might provide a starting point from which to model risk profiles that will facilitate improvements in drug therapy, in new drug design, and in other directions of interest. Figure 1 attempts to convey these ideas schematically.

Risk profiles of drug susceptibility would serve as stepping stones from the molecular foundations of pharmacogenetics to its long-term objectives. Next, we need to ask what might be some of the obstacles or challenges to advances in the design and construction of such profiles. Obviously, the construction of such risk profiles necessitates the collection of genomic data on a large scale. Although the number of pharmacogenetic traits that have been 
extensively characterized as of this writing probably considerably exceeds the estimate of a hundred traits made about 10 years ago, molecular studies indicate that such traits are usually associated with only a limited number of important variants. This raises the prospect that these genes and related haplotypes may be cataloged relatively quickly for many populations of interest. As the first stage of the Human Genome Project nears completion [Watson, 2000], the problem of collecting information on human pharmacogenetic diversity may presently be solved.

Additionally, for drug susceptibility profiling, we need to know the functional consequences of the human pharmacogenetic diversity. This means that the relationship of an individual's genotype (or haplotype) to his/her expressed phenotype must be determined. Pharmacologists usually think of an altered drug response as a consequence of a peculiarity in either the pharmacokinetics or the pharmacodynamics of the drug. Usually one suspects a defect in mechanisms that control the drug level (absorption, distribution, elimination) or of the receptor that mediates the response to the drug [see Weber, 1997, Chapter 2]. Although a defective pharmacokinetic mechanism is relatively easily identified, our present understanding of altered pathways or mechanisms at the molecular level that contribute to the pharmacodynamic (receptor-mediated) diversity of drug responses is relatively rudimentary. Thus, establishing genotype-phenotype relationships, particularly for the responses attributable to pharmacodynamic diversity, represents a major challenge, and one that is likely to require more than technical development for resolution.

However, even when the relationship of genotype (or haplotype) to a drug-susceptible phenotype is reasonably firm, the clinical application of pharmacogenetic knowledge to therapeutics is, with few exceptions, as yet unrealized. There are a number of reasons for limited carryover of this knowledge to therapeutics, which can best be illustrated by traits attributable to the human drug-metabolizing enzyme polymorphisms. These traits have been used to document the diversity in human drug response in different geographic and ethnic populations, and to identify the molecular basis of normal and altered drug responses. They have also shed light on the mechanisms that explain these responses, and to define the relationships between genotype and phenotype that together determine drug susceptibility.

Obviously, these traits have contributed immensely to the clarification of human variation in response to many drugs, and are expected to be important in new drug development. Despite having such an abundant collection of evidence, carryover to the clinical arena is still limited. Why? One reason is that much of the evidence collected during the 1960s through the 1980s concerned the polymorphic gene of interest in isolation from all other genes, and the evidence obtained was often limited to a few healthy individuals or small populations. Moreover, many of the earlier studies were designed to test proof of principle, that is, to test the relevance of genetic diversity to variation in human drug response, and were not designed to assess clinical end points [Weber, 1997].

Several additional limitations are evident from some the data obtained.

1. There is a lack of clear criteria for assessing the outcome of the polymorphism. Commonly, we see that one or more variables (such as genotypes, drug-metabolite ratios, urinary drug/metabolite patterns, or drug levels) may be used to assess outcome. Often the relationship between the variables and the clinical implications of such variation are unclear or incompletely defined.

2. There is a lack of information about genetic regulation of P450 drug-metabolizing enzymes by physiological and pathophysiological factors. Infections or inflammatory stimuli, or cytokines and interferons administered as therapeutic agents, as well as hormones and nutritional status can cause changes in the activities or expression of various forms of cytochrome (CYP450) enzymes in humans [reviewed in Morgan, 1997; Morgan et al., 1998]. These factors have the potential to affect, either adversely or favorably, the therapeutic or toxic effects of drugs. These effects are particularly crucial for drugs with a low therapeutic index. Although some important findings regarding the effects of these factors on drug levels of a few drugs have been known for many years [see Table 1 in Morgan, 1997], their systematic investigation has begun only recently. At the present time, very little is known of the effects of these factors on expression of Phase 1 (CYP450) or the Phase 2 drug-metabolizing enzyme polymorphisms.

3. There is a lack of adequate large-scale, high-throughput methodology for metabolic phenotyping. Knowledge of the genotype provides useful information about drug susceptibility, although this information alone is usually insufficient to predict the phenotype [Raimundo et al., 2000 and references therein]. However, genotyping complemented by phenotyping with probe substrates affords an alternative way of assessing the activity of specific drug-metabolizing enzymes in vivo. For some of the drug-metabolizing enzymes, including CYP1A2, 2E1, 3A, 2D6, and 2C19, and $N$-acetyltransferase (NAT2), the metabolic phenotype can be determined simultaneously with the aid of a cocktail of noninteracting substrates [Frye et al., 1997]. These techniques are employed now mainly in a research setting. The development of methodology for additional Phase 1 and Phase 2 enzymes applicable to large-scale, high-throughput analyses for therapeutic drug monitoring is urgently needed.

4. There is a lack of a clear dose (plasma concentration)response relationship for some disorders. For example, the genetic polymorphism of CYP2D6 has a profound 
effect on the pharmacokinetics of antidepressant and neuroleptic drugs, but there is a paucity of evidence for relationships between the plasma concentrations of these agents and their active metabolites and therapeutic response in depression [reviewed in Tucker, 2000]. It has not been possible to demonstrate convincingly that higher antidepressant drug plasma concentrations in CYP2D6-poor metabolizers are associated with greater amelioration of depression. The disconnection between the diagnosis, or severity, of depression and its pharmacokinetics, which is yet to be explained, presents an obstacle to the management of this disorder.

Nowadays, pharmacogenetics, or pharmacogenomics, is a hot topic at national and international scientific conferences. Recently, the Human Genome Project has received a great deal of attention at such conferences and in the popular press. The idea that genes contribute to drug response, and that drugs may be tailored to fit the genes of patients has also been treated informatively in the press [Altman, 1998; Carr, 1998; Wade, 1999, 2000; Lemonick, 2000]. Yet the belief that the effects of drugs on individuals are attributed primarily to the drug administered, and that the drug recipient is merely a passive bystander to these events, is much more widely held. People perceive an influence of heredity on health and disease, especially with respect to its effect on the occurrence of physical and mental traits in members of their families, but may be unaware of the harmful effects of medical drugs on genetically susceptible persons. It appears that we still have far to go in getting these points across to the general public and to explain the promise of pharmacogenetics to better medical care. Whether physicians and pharmacists in mainstream practice are sufficiently prepared to extend the benefits of individualized medicine to their patients raises another question. It is probably unrealistic to assume that the majority of these practitioners will respond effectively to such information without additional training and assistance.

\section{REFERENCES}

Altman LK. 1998. Drug shown to shrink tumors in type of breast cancer by targeting gene defect. New York Times, May 18, p A12.

Ballabio A. 1993. The rise and fall of positional cloning. Nat Genet 3:277-279.
Carr G. 1998. The alchemists. The Economist, February 21, p 1-18.

Curran ME. 1998. Potassium ion channels and human disease: phenotypes to drug targets. Curr Opin Biotechnol 9:565-572.

Drysdale CM, McGraw DW, Stack CB, Stephens JC, Judson RS, Nandabalan K, Arnold K, Ruano G, Liggett SB. 2000. Complex promoter and coding region $\beta 2$-adrenergic receptor haplotypes alter receptor expression and predict in vivo responsiveness. Proc Natl Acad Sci USA 97:10483-10488.

Frye RF, Matzke GR, Adedayo A, Porter JA, Branch RA. 1997. Validation of the five-drug "Pittsburgh cocktail" approach for assessment of selective regulation of drug metabolizing enzymes. Clin Pharmacol Ther 62:365-376.

Kwiatkowski RW, Lyamichev V, de Arruda M, Neri B. 1999. Clinical, genetic, and pharmacogenetic applications of the Invader assay. Mol Diagn 4:353-364.

Lefkowitz RJ. 2000. The superfamily of heptahelical receptors. Nat Cell Biol 2:E133-E136.

Lemonick MD. 2000. The genome is mapped. Now what? Time, July 3, p $24-29$.

Morgan ET. 1997. Regulation of cytochromes P450 during inflammation and infection. Drug Metab Rev 229:1129-1188.

Morgan ET, Sewer MB, Iber H, Gonzalez FJ, Lee Y-H, Tukey RH, Okino S, Vu T, Chen Y-H, Sidhu JS, Omiecinski CJ. 1998. Physiological and pathophysiological regulation of cytochrome P450. Drug Metab Dispos 26:1232-1240.

Raimundo S, Fischer J, Eichelbaum M, Griese E-U, Schwab M, Zanger UM. 2000. Elucidation of the genetic basis of the common 'intermediate metabolizer' phenotype for drug oxidation by CYP2D6. Pharmacogenetics 10:577-581.

Schafer AJ, Hawkins JR. 1998. DNA variation and the future of human genetics. Nat Biotechnol 16:33-39.

Tucker GT. 2000. Advances in understanding drug metabolism and its contribution to variability in patient responses. Ther Drug Monit 22:110-113

Wade N. 1999. Tailoring drugs to fit the genes. New York Times, April 20, p D9.

Wade N. 2000. Newfound protein touches off race for new therapies. New York Times, October 31, p D5.

Watson J. 2000. The double helix revisited. Time, July 3, p 30.

Weber WW. 1987. The acetylator genes and drug response. New York: Oxford University Press.

Weber WW. 1997. Pharmacogenetics. New York: Oxford University Press.

Weber WW. 2001. Pharmacogenetics-Receptors. In: Kalow W, Meyer UA, Tyndale R, editors. Pharmacogenomics. New York: Marcel Dekker.

Weber WW, Cronin MT. 2000. Pharmacogenetic testing. In: Meyers RA, editor. Encyclopedia of analytical chemistry. Chichester: Wiley. p $1506-1531$.

Accepted byT.A. Cebula 